\title{
What will it take to achieve virtual elimination of mother-to-child transmission of HIV? An assessment of current progress and future needs
}

\author{
Mary Mahy, ${ }^{1}$ John Stover, ${ }^{2}$ Karusa Kiragu, ${ }^{1}$ Chika Hayashi, ${ }^{3}$ Priscilla Akwara, ${ }^{4}$ \\ Chewe Luo, ${ }^{4}$ Karen Stanecki, ${ }^{1}$ Rene Ekpini, ${ }^{4}$ Nathan Shaffer ${ }^{3}$
}

\begin{abstract}
1 UNAIDS, Geneva, Switzerland ${ }^{2}$ Futures Institute, Glastonbury, Connecticut, USA

${ }^{3}$ World Health Organization,

Geneva, Switzerland

${ }^{4}$ UNICEF, New York, New York, USA
\end{abstract}

Correspondence to Dr Mahy, UNAIDS, 20 Avenue Appia, 1211 Geneva 27. Switzerland;

mahym@unaids.org

Accepted 7 October 2010

\section{ABSTRACT}

Background The number of HIV-positive pregnant women receiving antiretroviral drugs (ARVs) to prevent mother-to-child transmission (MTCT) of HIV has increased rapidly.

Objective To estimate the reduction in new child HIV infections resulting from prevention of MTCT (PMTCT) over the past decade. To project the potential impact of implementing the new WHO PMTCT guidelines between 2010 and 2015 and consider the efforts required to virtually eliminate MTCT, defined as $<5 \%$ transmission of HIV from mother to child, or $90 \%$ reduction of infections among young children by 2015 .

Methods Data from 25 countries with the largest numbers of HIV-positive pregnant women were used to create five scenarios to evaluate different PMTCT interventions. A demographic model, Spectrum, was used to estimate new child HIV infections as a measure of the impact of interventions.

Results Between 2000 and 2009 there was a 24\% reduction in the estimated annual number of new child infections in the 25 countries, of which about one-third occurred in 2009 alone. If these countries implement the new WHO PMTCT recommendations between 2010 and 2015, and provide more effective ARV prophylaxis or treatment to $90 \%$ of HIV-positive pregnant women, 1 million new child infections could be averted by 2015 . Reducing HIV incidence in reproductive age women, eliminating the current unmet need for family planning and limiting the duration of breastfeeding to 12 months (with ARV prophylaxis) could avert an additional 264000 infections, resulting in a total reduction of $79 \%$ of annual new child infections between 2009 and 2015, approaching but still missing the goal of virtual elimination of MTCT.

Discussion To achieve virtual elimination of new child infections PMTCT programmes must achieve high coverage of more effective ARV interventions and safer infant feeding practices. In addition, a comprehensive approach including meeting unmet family planning needs and reducing new HIV infections among reproductive age women will be required.

\section{INTRODUCTION}

In 2001, and again in 2006, national governments and their development partners committed themselves to reducing infant and child HIV infections through prevention of mother-to-child transmission (PMTCT) by $50 \%$ by $2010 .{ }^{1}{ }^{2}$ Recently, UNAIDS and partners have called for virtual elimination of MTCT of HIV. ${ }^{3}$ Virtual elimination includes an overarching goal of reducing the number of new child HIV infections by $90 \%$ between 2009 and 2015 and reducing MTCT to $<5 \%$. To prevent MTCT, the United Nations recommends a comprehensive four-pronged approach including (1) primary prevention of HIV infection among women of childbearing age; (2) preventing unintended pregnancies among women living with HIV; (3) preventing HIV transmission from women living with HIV to their infants and (4) providing appropriate treatment, care and support to mothers living with HIV and their children and families. ${ }^{4}$ Prongs 1,2 and 3 are necessary to reach the first target of reducing new child infections while only prong 3 will affect the second target to lower the transmission rate.

In countries where breastfeeding is common, the probability of transmission from mother to child without any antiretroviral drugs (ARVs) is approximately $20-45 \%$ : approximately $15-25 \%$ transmission during pregnancy and $5-20 \%$ transmission during breastfeeding. ${ }^{5}$ In developed countries, where all four prongs are well implemented and the most effective ARVs are provided to HIV-positive pregnant women with limited breastfeeding, the level of MTCT has decreased to below $2 \%{ }^{6-8}$ National measures of MTCT rates are not available in most developing countries because health systems cannot directly measure HIV infections resulting from vertical transmission in a systematic way.

In November 2009 WHO released Rapid Advice: Use of Antiretroviral Drugs for Treating Pregnant Women and Preventing HIV Infection in Infants and published revised full guidelines in July 2010. ${ }^{9-12}$ The new guidelines emphasise the importance of treating eligible HIV-positive pregnant women $(\mathrm{CD} 4 \leq 350)$ with lifelong antiretroviral therapy (ART) and recommend two equivalent options of highly effective prophylaxis to HIV-positive pregnant women who do not need ART for their own health. For the first time ARV prophylaxis to either the mother or child is also recommended during breastfeeding, in settings where breastfeeding is judged to be the safest infant feeding option. Option A starts treatment of women with zidovudine at 14 weeks of gestation or as soon as possible thereafter until delivery and the infant receives nevirapine throughout breastfeeding and until 1-week after exposure to breast milk has ended. Option B starts treatment of the mother with triple ARV prophylaxis from 14 weeks or as soon as possible thereafter and continues the 
maternal triple drug prophylaxis until 1-week after exposure to breast milk has ended. In 2010 WHO also released Guidelines on $H I V$ and Infant Feeding, which recommend exclusive breastfeeding for the first 6 months of life, and continued breastfeeding and complementary foods from months 6 to 12, when either the mother or baby is receiving ARVs. ${ }^{12}$

Effective prevention of MTCT requires a comprehensive approach that combines multiple interventions. While remarkable progress has been made in providing ARV regimens to HIV-positive pregnant women and to some extent in providing appropriate treatment, care and support to women living with HIV and their children, the other two prongs-preventing new HIV infections among reproductive age women and avoiding unwanted pregnancies among HIV-positive women-have had less support and success. In addition, although PMTCT is often the starting point for HIV-positive women to learn about their HIV status and enrol in HIV care and treatment services, postpartum follow-up of mother-infant pairs is often problematic for various reasons, including the lack of capacity of maternal and child health services to provide HIV testing or follow-up to HIV-exposed children. ${ }^{13} 14$

Here we describe the estimated reduction in new child HIV infections that has been achieved by providing PMTCT interventions in the 25 countries with the largest numbers of HIV-positive pregnant women. We project the potential number of child HIV infections that would be averted if $90 \%$ of HIV-positive pregnant women were provided with ARVs according to the new WHO guidelines, HIV incidence was reduced by $50 \%$ in reproductive age women and unwanted pregnancies were eliminated (by meeting all unmet family planning needs). In addition, while the new PMTCT ARV guidelines include prophylaxis during breastfeeding, we consider the impact of prolonged breastfeeding by estimating the number of new child infections that would be averted if the duration of breastfeeding while receiving prophylaxis were limited to 12 months. Although breastfeeding in many settings is safer than replacement feeding, it is important to recognise the impact of breastfeeding on HIV transmission.

\section{METHODS}

\section{Countries included in analysis}

The 25 countries with the largest number of HIV-positive pregnant women were chosen for this analysis based on their 2009 estimates reported in the 2010 Towards Universal Access report. ${ }^{15}$ These countries represent $91 \%$ of HIV-positive pregnant women in lower- and middle-income countries. The 25 countries are listed in table 1.

\section{Model}

In 2009 UNAIDS, WHO and partners conducted a series of training sessions to assist country teams to develop models to estimate and project their HIV epidemics using the Estimation and Projection Package $(\mathrm{EPP})^{16}$ and Spectrum ${ }^{17}$ software package. Country teams refined and improved the projections and incorporated the latest available data. The country files used for this analysis were submitted to UNAIDS by countries' teams in June 2010 and include data that were available at the end of 2009. Detailed descriptions of the methodologies for the models are described elsewhere ${ }^{1617}$ while a brief description is provided below.

Using HIV surveillance data from antenatal clinics and nationally representative household surveys, EPP fits epidemic curves for various geographical subregions using a maximumlikelihood approach. Incidence was estimated from prevalence over time, while adjusting for the number of people receiving antiretroviral therapy (ART). ${ }^{16}$ Finally, the subnational curves were aggregated to create national adult incidence curves.

HIV incidence among adults over time was transferred from EPP into Spectrum. Using demographic data from the UN Population Division's World Population Prospects 2008 revision, Spectrum projects men and women by age group over time through different stages of HIV infection (or non-infection).

National programme data were entered into Spectrum and parameters were modified based on available data. Data entered into the model related to calculating new child infections included the number of pregnant women receiving ARVs by regimen (single, dual and triple prophylaxis or ART for their own health) and type and duration of infant feeding by HIV-positive women. When countries do not have data on infant feeding practices among HIV-positive women, data from the general population were used to proxy infant feeding among HIV-positive women.

Spectrum uses the HIV incidence data estimated from EPP and creates age- and sex-specific HIV prevalence values. HIV prevalence was applied to the number of women in the age group and then age-specific fertility rates, adjusted to account for the lower fertility of HIV-positive women, were used to estimate the number of births to HIV-positive women by age of the mother. $^{18} 19$ See figure 1 .

MTCT was then calculated in two components: transmission during pregnancy and delivery (perinatal transmission) and transmission during breastfeeding. The assumed perinatal transmission rates for different ARV regimens are shown in table 2 .

HIV-exposed infants born HIV-negative (uninfected) have a monthly probability of HIV infection based on the infant feeding method and duration as well as ARV use during breastfeeding. The assumed monthly transmission rates during breastfeeding are shown in table 3 .

\section{Scenarios}

Spectrum was used to create five scenarios describing different situations and different interventions. The five scenarios include: 1. No PMTCT. No ARVs were provided to prevent MTCT at any point during the epidemic.

2. Base scenario. The 2009 programme was maintained through 2015-specifically the number of women and the regimen were kept constant from 2009 to 2015.

3. $90 \%$ on WHO guidelines. In 2010 the HIV-positive pregnant women currently receiving ARVs were switched to the 2010 WHO-recommended interventions (providing ART for women in need for their own health or starting women on ARV prophylaxis at 14 weeks and continuing ARVs throughout breastfeeding). The number of women receiving ARVs or ART was increased linearly to reach $90 \%$ by 2015.

4. Prongs 1,2,3. In addition to the changes in scenario 3, new HIV infections among reproductive age women were reduced by $50 \%$ and unmet need for family planning was eliminated. HIV incidence and unmet need were reduced linearly between 2010 and 2015.

5. Prongs 1,2,3 and reduced breastfeeding. In addition to prongs 1,2,3, median duration of breastfeeding among HIV-positive women was reduced to 12 months. See table 1 for country values.

Four comparisons were made between the scenarios to describe the impact of PMTCT.

\section{Comparison 1}

The no-PMTCT scenario described the situation if ARVs had never been available to prevent MTCT in the 25 countries. 


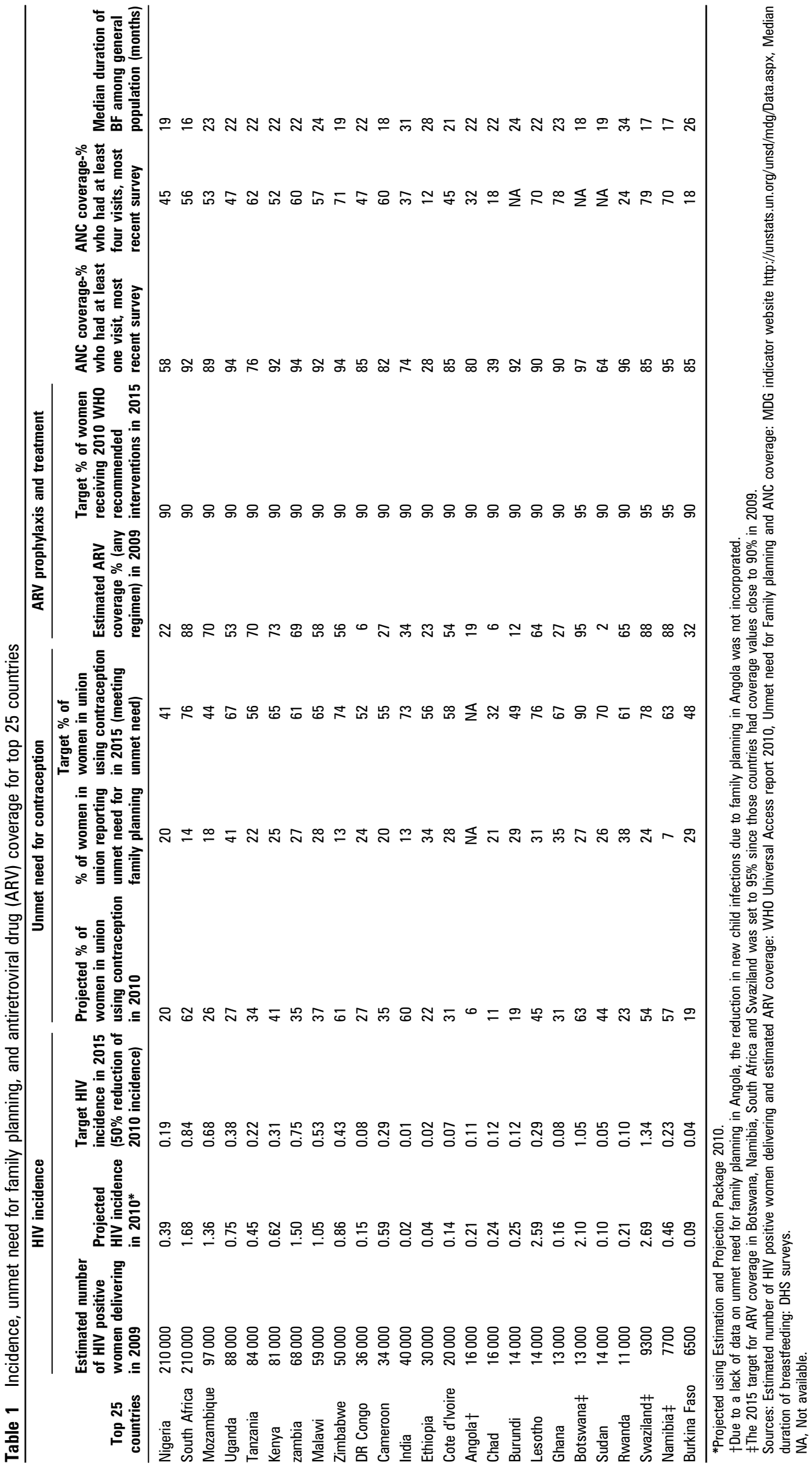




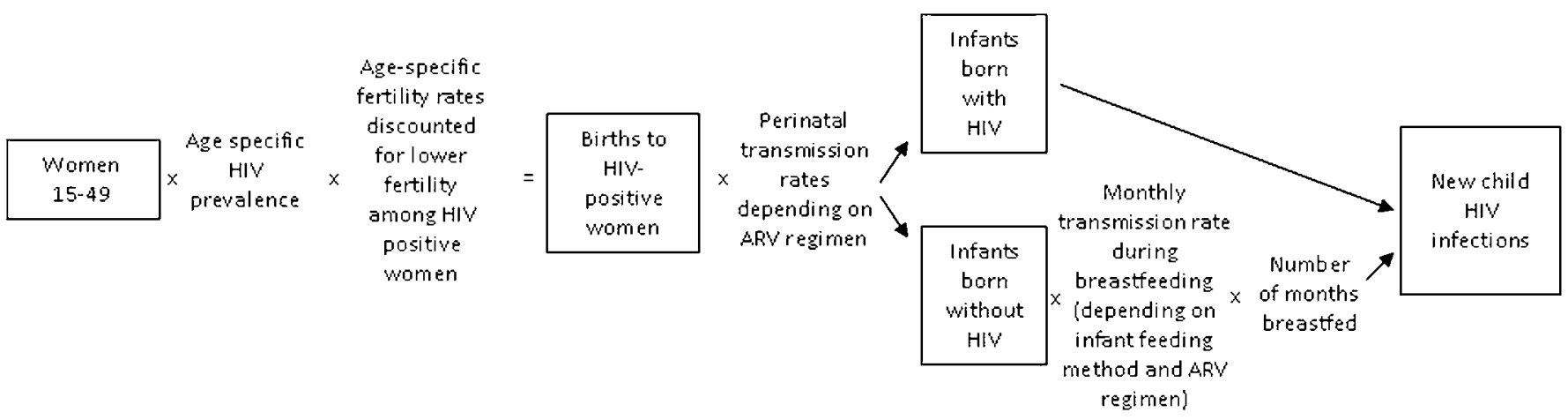

Figure 1 Summary of new child infections calculations. ARV, antiretroviral drug.

Comparing this scenario with existing coverage levels (the base scenario) provided an estimate of the impact of providing ARV prophylaxis, before the new WHO guidelines in the 25 countries.

The other comparisons were projections of what could be achieved in the coming years given different intervention levels.

\section{Comparison 2}

The base scenario was compared with the scenario where $90 \%$ of HIV-positive pregnant women received the 2010 WHO guidelines by 2015. All other elements of the epidemic remained the same following the epidemic curve projected from existing surveillance data.

\section{Comparison 3}

The base scenario was compared with the scenario in which prongs 1, 2 and 3 are implemented by 2015 .

\section{Comparison 4}

Again the base scenario was compared with a scenario where, between 2010 and 2015, prongs 1, 2 and 3 are implemented and the duration of breastfeeding was limited to 12 months.

\section{Unmet need for family planning}

To project unmet need for family planning (as in scenario 4), we used the family planning module available in the Spectrum computer package. This module allows the user to describe changes in contraceptive prevalence during the timeline of the projection. We linearly increased contraceptive prevalence from the projected value in 2010 (values of contraceptive prevalence are derived from household surveys and Spectrum then projects them forward in time) to the projected value plus unmet need in 2015. A measure of unmet need was available for 24 of the 25 countries in this analysis. No data on unmet need for family planning was available for Angola so the impact of increasing family planning efforts in Angola was not included in this analysis.

Unmet need for family planning is a common measure of access to family planning and is defined as the proportion of

Table 2 Assumed perinatal HIV transmission rates for different antiretroviral drug (ARV) regimens

\begin{tabular}{lll}
\hline ARV regimen & Percentage & Reference \\
\hline No ARV & 20 & 20 \\
Single-dose nevirapine & 11 & 2122 \\
Combination therapy using dual prophylaxis & 4 & 2324 \\
Combination therapy using triple prophylaxis ARV & 2 & 20 \\
Highly active antiretroviral treatment & 2 & \\
\hline
\end{tabular}

sexually active, fecund women in a union who wish to stop or postpone childbearing and are not currently using contraception. The measure can be interpreted as the increase in contraceptive prevalence rates if all women were able to fulfil their preferences. This measure was collected in household surveys of the general population. For PMTCT we were primarily interested in reducing the unintended pregnancies among HIV-positive women. The calculation of unmet need and its limitations have been described elsewhere. ${ }^{29} 30$

Table 1 presents the current levels of HIV incidence, unmet need for family planning and ARV coverage as well as the target values according to the scenarios described. Median duration of breastfeeding among all women by country and the proportion of pregnant women attending antenatal clinics is also provided to indicate the magnitude of change required to reach the targets set in the different scenarios.

The scenarios were implemented for each country individually and results were aggregated to create one estimate for the 25 countries with the largest numbers of HIV-positive pregnant women.

In this analysis we focused on new child infections as the outcome, although we recognised that many other outcomes could be quantified with other models and data. Future models should ideally estimate HIV-free child survival as the overall goal of interventions to prevent MTCT of HIV.

\section{RESULTS}

The scenarios described above were used to estimate the number of new child infections (mother-to-child vertical HIV infections), including perinatal infections as well as postpartum infections during breastfeeding.

\section{Comparison 1}

Between 2000 and 2009 an estimated 4.18 million children were infected with HIV in the 25 countries. If no ART or ARV prophylaxis had been provided to pregnant women approximately 4.45 million would have been infected. Cumulatively 269000 child HIV infections were prevented in the 25 countries

Table 3 Monthly HIV transmission rates during breastfeeding

\begin{tabular}{lll}
\hline Feeding method & Percentage & Reference \\
\hline Replacement feeding & 0 & \\
Mixed breastfeeding during months 1-6 & 1.5 & 2526 \\
Exclusive breastfeeding during months 1-6 & 0.75 & 2527 \\
Mixed breastfeeding 6-36 months & 0.75 & 2527 \\
Breastfeeding in the presence of maternal ART & 0.3 & 28 \\
or effective ARV prophylaxis & & \\
\hline ART, antiretroviral therapy; ARV, antiretroviral drug. &
\end{tabular}


by providing ARVs to HIV-positive pregnant women for prophylaxis or treatment. An estimated 89000 child infections, or a third of cumulative child infections, were averted in 2009 alone (there were an estimated 347000 new infections in the 25 countries in 2009). Comparing the estimated annual number of new child infections in 2000 with the annual number of new infections in 2009 shows a reduction of $24 \%$ among the 25 countries. See figure 2. (The slight decline in the no-PMTCT scenario in later years reflects changes in the number of HIV-positive pregnant women as incidence declined either owing to natural progression of HIV or owing to prevention efforts.)

\section{Comparison 2}

Projecting into the future, if current programmes were improved so that by 2015 90\% of HIV-positive pregnant women were provided with ART or highly effective ARV prophylaxis during pregnancy and throughout breastfeeding as currently recommended by $\mathrm{WHO}$, of the projected 2.1 million child infections between 2010 and 2015 approximately 1041000 new child infections would be averted (see figure 3). In the year 2015 alone, 229000 HIV infections would be averted among children. This is a $60 \%$ reduction in the annual number of new child infections between the year 2009 and 2015: a major step forward but still well below the goal of a $90 \%$ reduction. Even after reaching $90 \%$ of women with the new WHO recommended interventions through pregnancy and breastfeeding there would still be approximately 138000 new child infections in the year 2015 in the 25 countries. The MTCT rate in 2015 would be approximately $11 \%$ if $90 \%$ of women were reached with PMTCT services reflecting the WHO guidelines.

\section{Comparison 3}

If in addition to providing $90 \%$ of HIV-positive pregnant women with ART or more effective ARVs as recommended by WHO, new HIV infections among reproductive age women were reduced by $50 \%$ and unmet need for family planning for HIVpositive women was eliminated, the number of new HIV infections among children could be further reduced. Implementing these interventions simultaneously would avert approximately 1174000 (of 2.1 million) new child HIV infections between 2010 and 2015, resulting in a 73\% overall reduction in new child infections between the years 2009 and 2015 (see figure 3). As previously noted, including prongs 1 and 2 would not reduce the overall transmission rate, which would remain at approximately $11 \%$.

\section{Comparison 4}

The median duration of breastfeeding among women in these countries was 22 months. If, in addition to the above interventions, breastfeeding was limited to 12 months a total of 1306000 new child infections would be averted between 2010 and 2015; avoiding 264000 additional new child infections than the scenario with the 2010 WHO recommendations alone (see figure 3 ). This would result in a $79 \%$ reduction in annual new child infections in the 25 countries between 2009 and 2015. An estimated 72000 children would be infected with HIV through vertical transmission in 2015 (see figure 4). Assuming that only the women who receive ARVs (90\%) are counselled about reducing breastfeeding and the remaining $10 \%$ continue breastfeeding patterns that are reflected in the general population, the transmission rate would be approximately $8 \%$.

\section{LIMITATIONS}

This analysis was limited by the fact that the transmission rates in the model are based primarily on results from clinical studies which might not reflect the effectiveness of national programmes. These assumptions are being reviewed as new data become available. The model currently does not differentiate transmission rates by the CD4 level of the mother. Future versions of the model will attempt to incorporate this difference. In addition, data on the duration and methods of infant feeding are not readily available for HIV-positive women, and especially with the new recommendations of ARV prophylaxis during breastfeeding. This limits our ability to estimate HIV transmission during breastfeeding. Breastfeeding duration from the general population was the most appropriate available data to estimate duration of breastfeeding among HIV-positive women, although this is likely to be an overestimate. However, analysis from household surveys suggest little difference between the breastfeeding practices of HIV-positive women (who reported knowing their HIV status) and HIV-negative women. ${ }^{31}$ Finally, the models rely on programme data from the countries on PMTCT coverage and ART coverage. These data can be of varying quality ${ }^{32}$ The data used for this analysis underwent a global reconciliation process comparing data reported from countries through multiple reporting mechanisms.
Figure 2 New HIV infections through mother-to-child transmission (MTCT) if antiretroviral drugs (ARVs) were not available versus reported ARV coverage, 25 countries, 2000-9. PMTCT, prevention of MTCT.

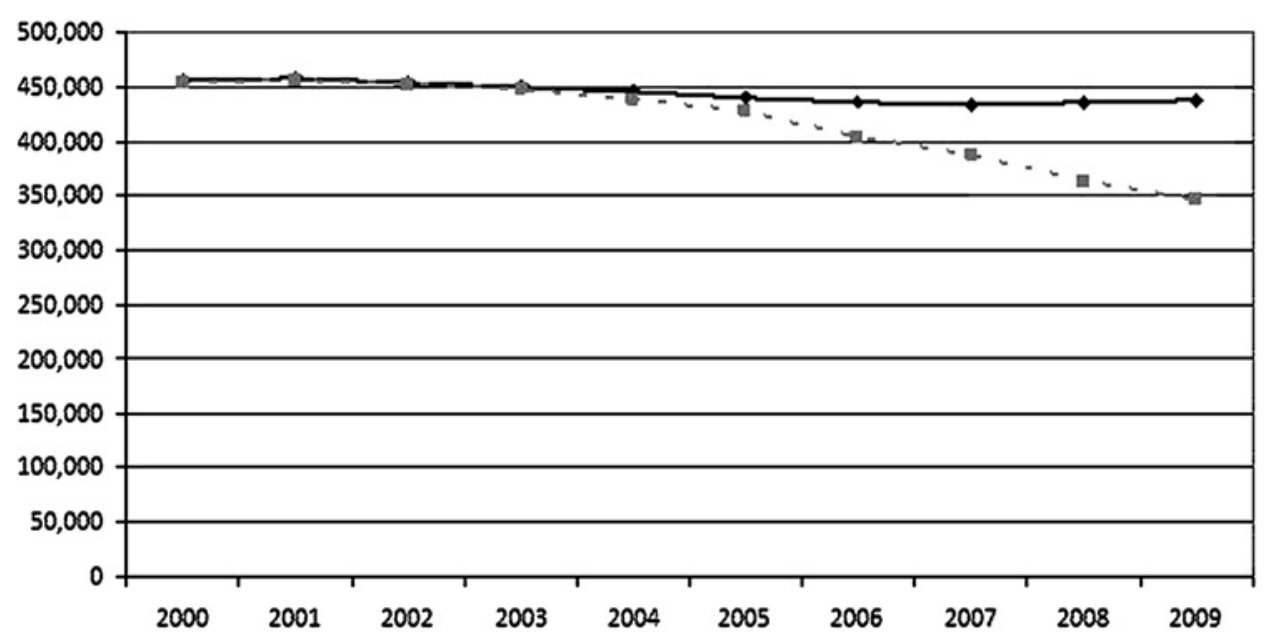


Figure 3 New HIV infections through mother-to-child transmission (MTCT) for different scenarios, 25 countries, 2009-15. ARV, antiretroviral drug; PMTCT, prevention of MTCT.

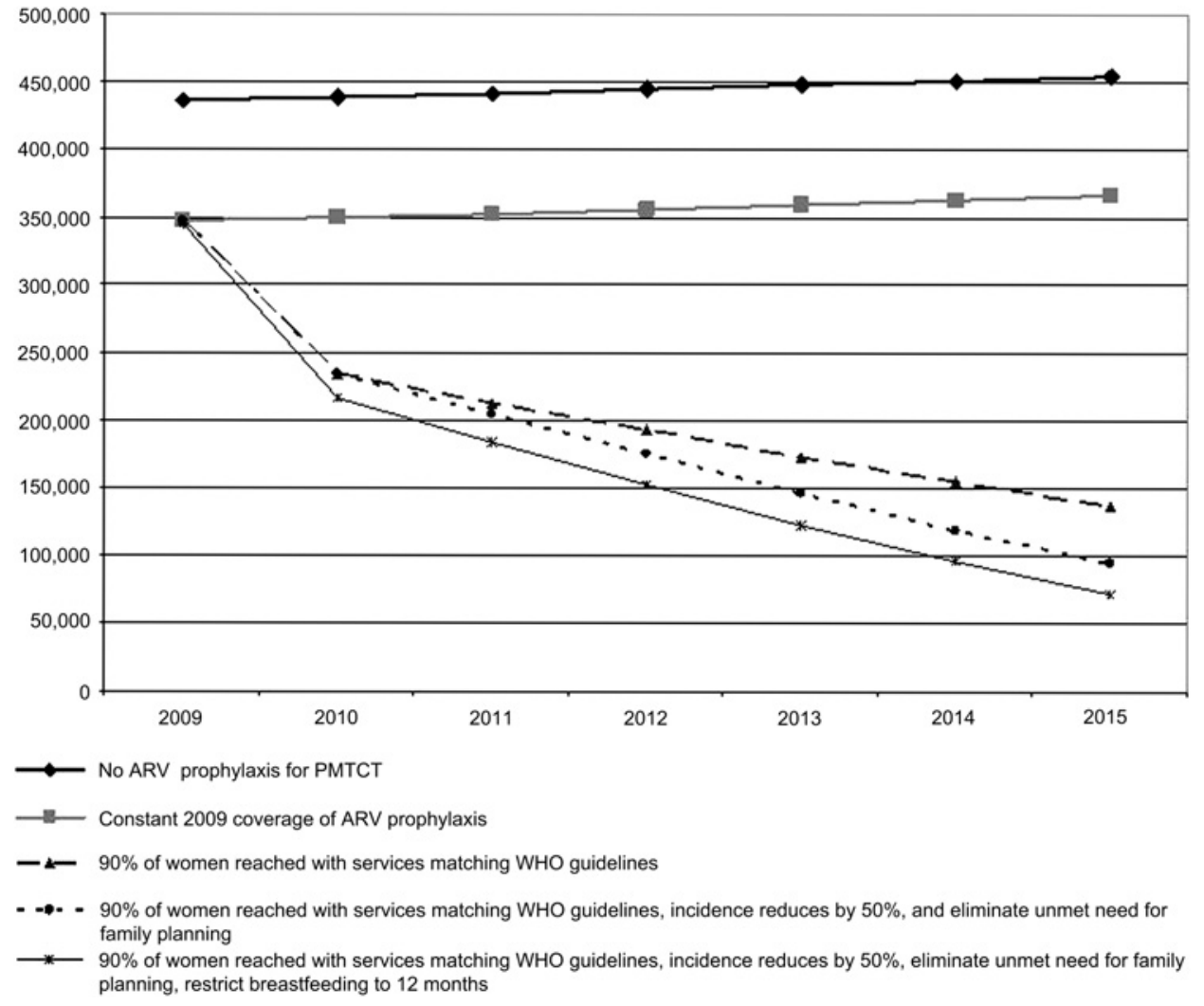

\section{DISCUSSION}

Between 2000, when ARV prophylaxis for MTCT was initiated in developing countries, and 2009 approximately 269000 new child infections were averted in the 25 highest burden countries analysed here (representing approximately $91 \%$ of HIV-positive pregnant women in low- and middle-income countries). There was an estimated $24 \%$ reduction in the number of annual new child infections between 2000 and 2009 as a result of PMTCT ARV interventions. Targets are being set at global and country level to reduce new infections due to MTCT by $90 \%$ over the coming years. In 2009 there were an estimated 347000 new child infections in the 25 countries. A $90 \%$ reduction in new child infections would require fewer than 34700 new infections in 2015 in the 25 countries.

Our study suggests there will still be 72000 new child infections in 2015 in the 25 highest burden countries if we rapidly scale up PMTCT interventions (reaching $90 \%$ of women with ART or highly effective ARV prophylaxis through delivery and breastfeeding as currently recommended by WHO, halving new HIV infections among reproductive age women, eliminating

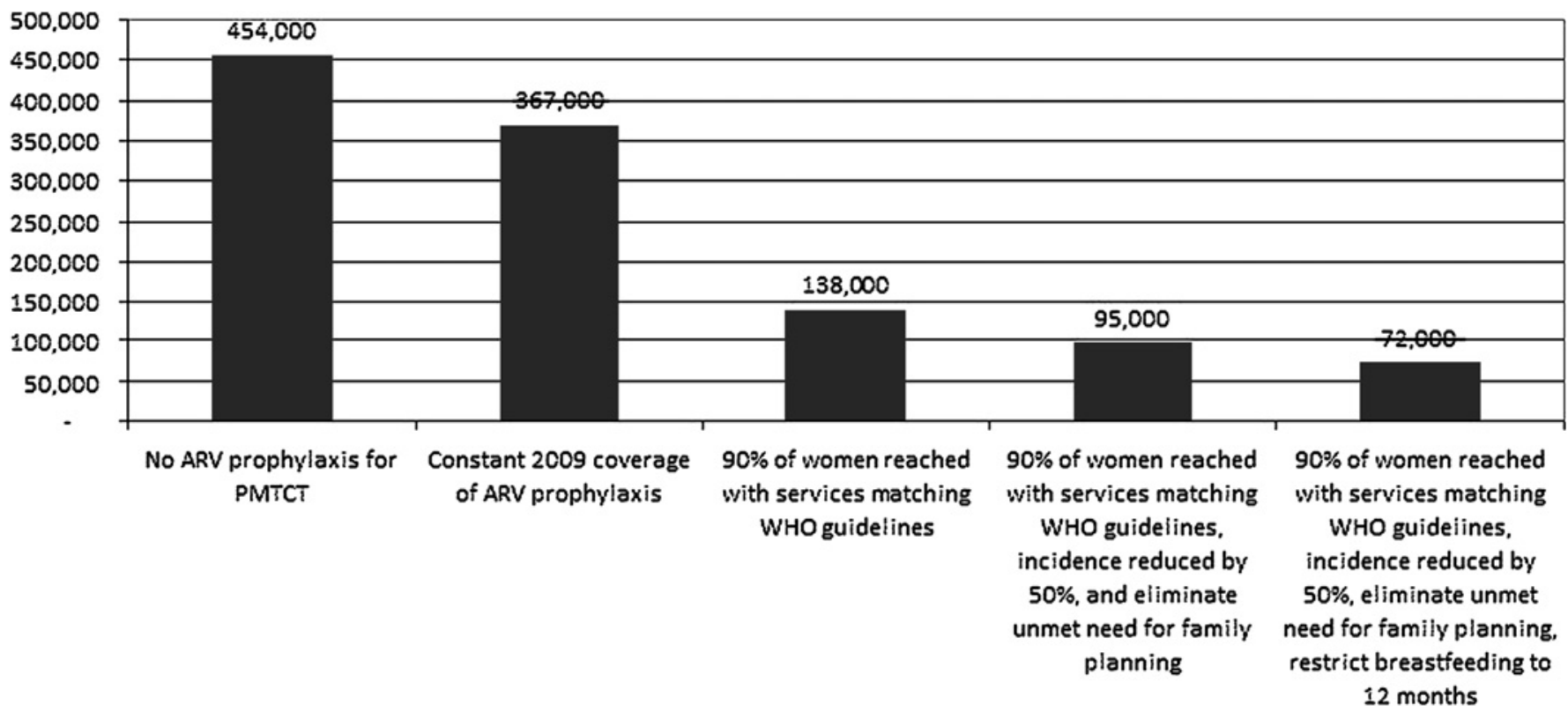

Figure 4 New HIV infections through mother-to-child transmission (MTCT), 25 countries, in the year 2015. ARV, antiretroviral drug; PMTCT, prevention of MTCT. 
unmet need for contraception and reducing the duration of breastfeeding to 12 months): a reduction of $79 \%$ in annual new child infections between 2009 and 2015. Under these assumptions, the rate of MTCT would be $8 \%$. Both projections represent significant progress and reductions in new child infections, but still fall short of the definition of virtual elimination: a $90 \%$ reduction in new child infections or $<5 \%$ transmission rate.

Reaching the ambitious new targets of virtual elimination will require significant resources and commitment, especially in specific high-burden countries with major barriers. For example Ethiopia, with very low antenatal care registration will need to increase attendance at antenatal clinics from $28 \%$ to at least $90 \%$. Lesotho, with high HIV incidence in pregnant women, will need to reduce incidence rates from $2.6 \%$ to $1.3 \%$. Uganda will need to increase the availability of family planning so that contraceptive use will increase from $27 \%$ to $67 \%$ (see table 1). On the other hand, 20 of the 25 countries have current antenatal care coverage (at least one visit) $>75 \%$ making it feasible, with substantial effort, for these countries to reach most women with PMTCT services. However, none of the 25 countries currently reach $90 \%$ of women with the recommended four antenatal care visits (table 1), suggesting additional efforts are needed.

This analysis shows the importance of moving rapidly to the new, more effective ARV interventions recommended in the 2010 WHO guidelines, but also shows some of the limitations of achieving virtual elimination, even with the new interventions. Our analysis suggests that coverage with highly effective ARV interventions will need to be $>90 \%$ (probably >95\%) and that prolonged breastfeeding beyond 12 months, even with ARVs, will increase the risk of new infections. To reach an approximate $80 \%$ reduction in new child infections, the duration of breastfeeding with ARVs had to be limited to 12 months. Although one recent clinical study found extremely low monthly transmission rates among breastfeeding infants, the median monthly transmission rate over 10 clinical trials with ARV interventions between 2007 and 2010 was $0.3 \%{ }^{28}{ }^{33}$ Depending on the duration of breastfeeding this transmission can accumulate to significantly increase the overall transmission rate. For example, the probability of transmission during 12 months of breastfeeding is $3.6 \%$; adding the perinatal transmission rate with $100 \%$ of women on the 2010 WHO guideline interventions results in an overall transmission rate of $5.6 \%$.

Given our current understanding of infant feeding practices in these 25 countries, reaching the virtual elimination goal of $<5 \%$ transmission rate may not be possible by providing ARVs alone. Even if $100 \%$ of HIV-positive pregnant women were provided with the $2010 \mathrm{WHO}$ guideline interventions, a transmission rate of $<5 \%$ would only be possible if infant feeding were safer. In Botswana, for example, the majority of HIV-positive women choose to use replacement feed and vertical transmission is estimated to be $3.7 \% .{ }^{34}$ However, replacement feeding is not a simple intervention and can lead to higher infant mortality in some settings. ${ }^{35}$ More effort is needed to ensure that mothers are fully informed about the risks and benefits of breastfeeding especially over long durations, as recommended in the WHO Guidelines on HIV and Infant Feeding. ${ }^{12}$ Even when women or children are receiving ARVs to reduce transmission there is still a probability of infection for each month that the child is exposed to breast milk. As countries begin to scale up the new WHO guidelines, including safer breastfeeding practices with ARVs, new programme data are needed on the effectiveness of ARV interventions during breastfeeding and the duration of breastfeeding among HIV-positive women.
Caution is needed when interpreting the additional impact of the different interventions. The impact of the interventions depends on the order in which they are implemented. For example, if unmet need for family planning was eliminated before ARVs were provided we would estimate that family planning had a much larger impact on reducing new child infections. We could also hypothesise that by eliminating unmet need for family planning, women living with HIV would have the children they want when they want them. This also means that the pregnancies at which PMTCT ARVs are directed would be largely wanted pregnancies. Women with such pregnancies might have a higher motivation to adhere to PMTCT regimens, a crucial factor given the earlier start and longer duration of ARVs recommended by the 2010 WHO guidelines. In practice, the interventions would not be implemented individually thus we have not estimated the individual impact of interventions here.

The PMTCT interventions described here can dramatically reduce new child HIV infections. However, these interventions have many additional benefits beyond reducing new child HIV infections. By eliminating unmet need for family planning and reducing HIV incidence by $50 \%$ in the top 25 countries $50 \%$ fewer pregnant women would be HIV positive, approximately 36 million (cumulative between 2010 and 2015) unwanted births would be avoided among all women, and 1209000 fewer HIV-positive pregnant women would require ARV prophylaxis.

Much of the global effort for PMTCT has been focused on prong 3, provision of ARV prophylaxis. Additional efforts are needed on prong 1 , primary prevention, and prong 2 , meeting the unmet need for family planning among women living with HIV to reduce child infections. Meeting the current unmet need for family planning at a country level will require considerable effort. Yet countries are more likely to reach virtual elimination if they raise demand for family planning, and strengthen programmes to meet this demand. The measure of unmet need used in this analysis is based on all women and is thus likely to be an underestimate of unmet need for HIV-positive women. ${ }^{36}$ Thus the impact of meeting unmet need for family planning might be larger than predicted in this analysis. If more women are reached with testing and counselling the demand for family planning might be even greater.

Meeting global and country-specific targets for virtual elimination of MTCT of HIV will take an enormous amount of effort and resources from government, civil society and donors. The financial requirements should be carefully considered to help countries plan effective, efficient interventions to reach these goals. Clear progress has been made in the first 10 years of this global effort. With the scale-up of more effective ARV

\section{Key messages}

- Progress has been made in the first 10 years of prevention of mother-to-child transmission but significant resources are required to reach virtual elimination by 2015 .

- Even after providing the WHO recommended antiretroviral drug prophylaxis or antiretroviral treatment to $90 \%$ of HIVpositive pregnant women, prolonged breastfeeding will results in continued new child infections.

- Strengthening programmes to improve access to family planning and reduce new HIV infections will be critical to reach the virtual elimination targets. 
interventions, together with a comprehensive approach to reduce new infections in pregnant women and meet family planning needs, rapid progress can be made towards virtual elimination.

Competing interests None.

Contributors MM conceptualised and drafted the manuscript and data analysis; JS developed Spectrum computer package and drafted parts of the manuscript; KK conceptualised and commented on the manuscript; $\mathrm{CH}$, PA, CL, RE provided comments on the manuscript; KS supervised the country estimates process and provided comments on the manuscript; NS drafted portions and commented on manuscript.

Provenance and peer review Not commissioned; externally peer reviewed.

\section{REFERENCES}

1. United Nations. Declaration of Commitment on HIV/AIDS. New York, 2001. http:// data.unaids.org/publications/irc-pub03/aidsdeclaration_en.pdf laccessed 9 Aug 2010).

2. United Nations. Political declaration on HIV/AIDS: resolution adopted by the General Assembly. New York: United Nations, 2006. http://data.unaids.org/pub/ Report/2006/20060615 hlm politicaldeclaration ares60262 en.pdf laccessed 9 Aug 2010).

3. Michel S, Tedstrom J. The business of eliminating mother-to-Child HIV transmission. The Huffington Post 2010.

4. World Health Organization, UNICEF, Interagency task team on Prevention of HIV infection in Pregnant Women Mothers and their Children. Guidance on global scale-up of the prevention of mother to child transmission of HIV Geneva: World Health Organization, 2007. http://www.unicef.org/aids/files/ PMTCT enWEBNov26.pdf (accessed 7 May 2010).

5. De Cock KM, Fowler MG, Mercier E, et al. Prevention of mother-to-child HIV transmission in resource-poor countries: translating research into policy and practice. JAMA 2000:283:1175-82.

6. Townsend CL, Cortina-Borja M, Peckham CS, et al. Low rates of mother-to-child transmission of HIV following effective pregnancy interventions in the United Kingdom and Ireland, 2000-2006. AIDS 2008:22:973-81.

7. McKenna MT, Hu X. Recent trends in the incidence and morbidity that are associated with perinatal human immunodeficiency virus infection in the United States. Am J Obstet Gynecol 2007:197:S10-S16.

8. European Collaborative Study. The mother-to-child HIV transmission epidemic in Europe: evolving in the East and established in the West. AIDS 2006:20:1419-27.

9. World Health Organization. HIV and Infant Feeding: Revised principles and recommendations. Geneva: World Health Organization, 2009. http://www.who.int/ hiv/pub/paediatric/advice/en (accessed 1 Jun 2010).

10. World Health Organization. Antiretroviral drugs for treating pregnant women and preventing HIV infection in infants: Towards universal access Recommendations for a public health approach. Geneva: World Health Organization, 2010. http://www.who.int/hiv/pub/mtct/arv_guidelines_mtct.pdf (accessed 9 Aug 2010)

11. World Health Organization. Rapid advice: use of antiretroviral drugs for treating pregnant women and preventing HIV infection in infants. Geneva: World Health Organization, 2009. http://www.who.int/hiv/pub/mtct/advice/en (accessed 7 May 2010)

12. World Health Organization. Guidelines on HIV and infant feeding 2010 Geneva: World Health Organization, 2010. http://whqlibdoc.who.int/publications/2010/ 9789241599535 eng.pdf (accessed 9 Aug 2010).

13. Sherman G, Jones S, Coovadia A, et al. PMTCT from research to reality-results from a routine service. S Afr Med J 2004;94:289-92.

14. Nyandiko WM, Otieno-Nyunya B, Musick B, et al. Outcomes of HIV-Exposed Children in Western Kenya: Efficacy of Prevention of Mother to Child Transmission in a Resource-Constrained Setting. JAIDS 2010;54:42-50.
15. World Health Organization, UNICEF, UNAIDS. Towards universal access. Geneva: World Health Organization, 2010. http://www.who.int/hiv/pub/ 2010progressreport/report/en/index.html (accessed 30 Sep 2010).

16. Brown T, Bao L, Raftery AE, et al. Modelling HIV epidemics in the antiretroviral era: the UNAIDS Estimation and Projection package 2009. STI Supplement 2010; 86(Suppl 2):ii3-10.

17. Stover J, Johnson P, Hallett T, et al. The Spectrum projection package: improvements in estimating incidence by age and sex, mother-to-child transmission HIV progression in children and double orphans. STI Supplement 2010;86(Suppl 2): ii16-21.

18. Stover J. Aim a computer program for making HIVIAIDS projectinos and examining the demographic and social impacts of AIDS. Glastonbury, CT: Futures Institute, 2009. http://data.unaids.org/pub/Manual/2009/20090414_aim_manual_2009_en. pdf (accessed 15 Sep 2010)

19. Lewis JJ, Ronsmans C, Ezeh A, et al. The population impact of HIV on fertility in sub-Saharan Africa. AIDS 2004; 18:S35-43.

20. World Health Organization, UNICEF. HIV Transmission Through Breastfeeding: A Review of Available Evidence 2007 Update. Geneva: World Health Organization, 2007. http://whqlibdoc.who.int/publications/2008/9789241596596_eng.pdf (accessed 11 May 2010).

21. Jackson JB, Musoke P, Fleming T, et al. Intrapartum and neonatal single-dose nevirapine compared with zidovudine for prevention of mother-to-child transmission of HIV-1 in Kampala, Uganda: 18-month follow-up of the HIVNET 012 randomised trial. Lancet 2003;362:859-68.

22. Moodley D, Moodley J, Coovadia H, et al. A multicenter randomized controlled tria of nevirapine versus a combination of zidovudine and lamivudine to reduce intrapartum and early postpartum mother-to-child transmission of human immunodeficiency virus type 1. J Infect Dis 2003:187:725-35.

23. Lallemant M, Jourdain $\mathrm{G}$, Le Coeur $\mathrm{S}$, et al. Single-dose perinatal nevirapine plus standard zidovudine to prevent mother-to-child transmission of HIV-1 in Thailand N Engl J Med 2004;351:217-28.

24. ANRS 1201/1202 Ditrame PLUS Study Group. Field efficacy of zidovudine, lamivudine and single-dose nevirapine to prevent peripartum HIV transmission. AIDS 2005: 19:309-18

25. Iliff PJ, Piwoz EG, Tavengwa NV, et al. Early exclusive breastfeeding reduces the risk of postnatal HIV-1 transmission and increases HIV-free survival. AIDS 2005;19:699-708.

26. Breastfeeding and HIV International Transmission Study. Late Postnatal Transmission of HIV-1 in Breast-Fed Children: An Individual Patient Data Meta-Analysis. J Infect Dis 2004;189:2154-66.

27. Becquet $\mathbf{R}$, Bland $R$, Leroy $V$, et al. Duration, pattern of breastfeeding and postnatal transmission of HIV: pooled analysis of individual data from West and South African Cohorts. PLOS ONE 2009;4:e7397.

28. Mofenson Lynne M. Prevention in neglected subpopulations: prevention of mother-to-child transmission of HIV infection. Clin Infect Dis 2010;50:S130-48.

29. Westoff C. Unmet need at the end of the century: ORC Macro, 2001. http://www. measuredhs.com/pubs/pdf/CR1/C1.pdf (accessed 10 May 2010).

30. Westoff C. Desired number of children. Calverton, Maryland: ICF Macro, 2010. http://www.measuredhs.com/pubs/pdf/CR25/CR25.pdf (accessed 7 May 2009).

31. Bradley S, Mishra V. HIV and Nutrition among women in Sub-Saharan Africa. Calverton, Maryland: ICF Macro, 2008. http://www.measuredhs.com/pubs/pdf/ AS16/AS16.pdf (accessed 9 Aug 2010).

32. World Health Organization, UNAIDS, UNICEF. Towards universal access: scaling up priority HIVIAIDS interventions in the health sector, Progress Report 2009. Geneva: World Health Organization, 2009. http://www.who.int/hiv/pub/2009progressreport/en (accessed 6 May 2010).

33. Shapiro RL, Hughes MD, Ogwu A, et al. Antiretroviral regimens in pregnancy and breast-feeding in Botswana. N Engl J Med 2010;362:2282-94.

34. Stover J, Fidzani B, Molomo BC, et al. Estimated HIV trends and program effects in Botswana. PLOS ONE 2008;3:e3729.

35. Creek TL, Kim A, Lu L, et al. Hospitalization and mortality among primarily nonbreastfed children during a large outbreak of diarrhea and malnutrition in Botswana, 2006. JAIDS 2010;53:14-19.

36. Adair T. Desire for children and unmet need for contraception among HIV-positive Women in Lesotho. Calverton, MD: Macro International Inc, 2007. http://www. measuredhs.com/pubs/pdf/WP32/WP32.pdf (accessed 30 Jun 2010). 